\title{
The Worcestershire Prostate Cancer Database
}

Worcestershire has a population of over 500,000 people. There are 600 men newly diagnosed with prostate cancer in Worcestershire every year. This translates into 300 post surgery and around 500 post radiotherapy. Taking this into account a new pathway for holistic survivorship care as developed (Fig. 76.1). Once patients with prostate cancer have entered the 'survivorship phase,' they offered the option of entering into the Prostate Cancer Follow-up Database. Patients who have initial therapy with curative intent for organ confined disease are identified by a specialist nurse and invited to join the newly developed programme. Suitable patients will be identified via urology clinics held at Worcestershire Acute Hospitals. Patients must be 2 years post radical prostatectomy with an unrecordable PSA reading, 3 years post external beam radiotherapy with no metabolic relapse [1] or brachytherapy. Recurrence will be monitored via the PSA. Patients are discharged from clinic and are entered into a password protected database by the specialist nurse. The specialist nurse act as the patients' keyworker. This means that should the patient have any queries, the specialist nurse is the first point of contact. This database draws in information from pathological and clinical systems. This database can generate alerts if PSA results are elevated. Patients would be brought back to the clinic by the specialist nurse if; the PSA is elevated, with symptoms/signs of recurrence, adverse effects of treatment or at the patient's request.

\section{Reference}

1. Roach M 3rd, Hanks G, Thames H Jr, Schellhammer P, Shipley WU, Sokol GH, Sandler H. Defining biochemical failure following radiotherapy with or without hormonal therapy in men with clinically localized prostate cancer: recommendations of the RTOG-ASTRO Phoenix Consensus Conference. Int J Radiat Oncol Biol Phys. 2006;65(4):965-74. 